\title{
Clinical significance of ADAM29 promoting the invasion and growth of gastric cancer cells in vitro
}

\author{
HONGBING $\mathrm{CHEN}^{1 *}$ and SIPING WANG ${ }^{2 *}$ \\ ${ }^{1}$ Department of Gastrointenstinal Surgery, The Affiliated Yantai Yuhuangding Hospital of Qingdao University, Yantai, \\ Shandong 264000; ${ }^{2}$ Department of Emergency, Yantaishan Hospital, Yantai, Shandong 264001, P.R. China
}

Received October 14, 2017; Accepted April 6, 2018

DOI: $10.3892 / \mathrm{ol} .2018 .8838$

\begin{abstract}
ADAM metallopeptidase domain 29 (ADAM29) belongs to the ADAM family, is a type I integral membrane protein and secrets a glycoprotein that mediates cell-cell and cell-matrix interaction. Aberrant expression of ADAM29 is involved in a number of physiological processes diversification. The purpose of the present study was to investigate the expression and biological effect of ADAM29 in human gastric cancer (GC) specimens and cell lines in vitro. The expression of ADAM29 was examined in 83 GC samples and 25 adjacent normal gastric tissues using quantitative reverse transcriptase-quantitative polymerase chain reaction and immunohistochemistry. The association between ADAM29 expression and cellular function of GC cells was assessed in vitro. The ADAM29 mRNA levels were significantly elevated in GC tissues compared with paracancerous tissues. Increased levels of ADAM29 were associated with high-grade staging and high Tumor-Node-Metastasis stages. Kaplan-Meier survival curves demonstrated that patients with GC and low ADAM29 transcript levels exhibited longer overall survival $(\mathrm{OS})(\mathrm{P}<0.01)$ and progression-free survival (PFS) time $(\mathrm{P}<0.01)$ compared with patients with high ADAM29 expression levels. ADAM29 significantly promoted the proliferation, migration and invasion of GC cells in vitro when overexpressed in MGC803 cells and knocked down in AGS cells. ADAM29 was increased in GC and the elevated expression of ADAM29 was associated with a poor survival rate of patients. ADAM29 may become a prognostic factor and therapeutic candidate for human GC.
\end{abstract}

Correspondence to: Dr Hongbing Chen, Department of Gastrointenstinal Surgery, The Affiliated Yantai Yuhuangding Hospital of Qingdao University, 20 East Yuhuangding Road, Yantai, Shandong 264000, P.R. China

E-mail: ytyhdyy1108@126.com

${ }^{*}$ Contributed equally

Key words: ADAM family, ADAM metallopeptidase domain 29, gastric cancer, metastasis

\section{Introduction}

Gastric cancer (GC) is associated with high morbidity and mortality, and remains the third leading cause of cancer mortality globally and most prevalent cancer in East Asia $(1,2)$. The majority of patients are diagnosed at an advanced stage, during which curative surgery is necessary with extended lymphadenectomy. Endoscopic screening and laparoscopic distal gastrectomy are effective treatments for advanced GC (3); however, the 5-year overall survival rate for patients with GC remains poor, with a $<20 \%$ survival rate worldwide due to distant and high frequency metastasis in 2017 (4). Therefore, it is of great importance to investigate the underlying mechanisms of GC distant metastasis, and to identify effective diagnostic biomarkers of and treatments for GC.

Tumor cells disseminate from the primary tumor to other locations in the body, and rely on modifications in signaling pathways to affect proliferation, migration and invasion. An improved understanding of the molecular mechanisms underlying metastasis may prolong survival and maintain quality of life by relieving tumor-associated symptoms. The tumor microenvironment is a pivotal factor in tumorigenesis and tumor metastasis for coordinating the morphological transformation of cancer cells (5). Studies have highlighted the important role of the ADAM family, which may be associated with tumor microenvironment (6-8).

The ADAM proteins are a family of membrane-anchored glycoproteins mediating cell-matrix interactions (9). They are essential and are singularly active during the reprogramming of pluripotent stem cells (10). Their protease and adhesion domains are responsible for cell fusion (11), adhesion and signaling $(9,12)$. Previously, several studies identified aberrant expression of ADAMs in human cancer tissues $(13,14)$, which may be associated with carcinogenesis. For example, the overexpression of ADAM metallopeptidase domain 12 (ADAM12) contributed to increased cell proliferation through regulating the expression of phosphorylated (p)-protein kinase B and p-glycogen synthase kinase- $3 \beta$ in non-Hodgkin's lymphoma (15). ADAM17 promoted growth, migration and invasion in gastric carcinoma cells via the transforming growth factor (TGF)- $\beta /$ Smad pathway (16). Furthermore, ADAM17 was demonstrated to be a potential therapeutic target for GC and serve a key role in GC progression (16). 
ADAM29 is a member of the ADAM family, located on human chromosome 4q34 (17). Previous studies indicated that ADAM29 was involved in cancer development and progression (18-21). ADAM29 is frequently mutated in several tumor types, including breast and lung cancer, in the form of 4 missense mutations, all located in the same residue (p.Q814H), potentially forming a mini-hotspot (18). A previous study demonstrated that the mutation of ADAM29 in melanoma affected melanoma cell adherence to specific extracellular matrix proteins, and in a number of cases promoted the migratory ability of the cells (19). Studies regarding ADAM29 in breast cancer have also been conducted: For example, genome-wide studies indicated ADAM29 was a susceptible locus and a risk factor for breast cancer (20). In addition, a previous study detected an increased expression level of ADAM29 in breast cancer tissues compared with normal tissues (21). ADAM29 overexpression and its mutation in various domains affected the migration, growth and invasion of breast cancer cells in vitro (22). The roles of ADAM29 in other malignant tumor types were also investigated: ADAM29 was demonstrated to exhibit a high mutation rate in several cancer types, including colorectal cancer $(23,24)$, esophageal cancer (18) and melanoma (19). Previous data demonstrated that ADAM29 was upregulated in the MKN45 gastric carcinoma cell line with Mucin 1, cell surface associated downregulated clones, which decreased the rate of cell proliferation (25); however, the molecular mechanism underlying ADAM29 and its biological function in GC remains unknown.

In the present study, an investigation into the expression of ADAM29 in tumor and paracancerous tissues $(2 \mathrm{~cm}$ adjacent from the tumor) of patients with $\mathrm{GC}$, and the association between the expression level of this molecule with the clinical outcomes of patients, was conducted. Additionally, the biological functions of ADAM29 in human GC AGS and MGC803 cell lines were examined following the manipulation of ADAM29 expression in vitro.

\section{Materials and methods}

Human GC specimens. Human gastric tissue samples were collected from 83 patients aged 53 to 87 years with GC (comprised of 31 females, mean age 68.24 \pm 8.92 years; 52 males, mean age $72.34 \pm 9.34$ years) who had received curative resection, and immediately stored at $-80^{\circ} \mathrm{C}$ for further use. Inclusion criteria for patient selection were: i) Histological or cytological confirmation of GC; ii) Positron emission tomography-computed tomography revealed no clinically positive nodes; iii) patients were $\geq 18$ years of age. Exclusion criteria included: i) Inconsistent results of qPCR of 3 repeated experiments; ii) or if patients had previously been diagnosed with severe vascular, cerebrovascular or heart disease. The samples included gastric tumor tissues $(n=83)$ and normal paracancerous paired tissues $(n=25)$ an equivalent number of paracancerous tissues could not be obtained due to limitations of tissue size, and were collected from patients in Yantai Yuhuangding Hospital from January 2010 to December 2017. The number and sample classifications of specimens were verified by two pathologists from Yantai Yuhuangding Hospital (Yantai, China), were confirmed to be free from tumor deposits. The present study was executed accordingly under the protocol approved by the Institutional Review Board and Research Ethical Committee of the Affiliated Yantai Yuhuangding Hospital of Qingdao University (Yantai, China). Written informed consent was obtained from all patients. Clinicopathological factors, including, sex, tumor stage, Tumor-Node-Metastasis staging and lymph node metastasis (26), were analyzed and are presented in Table I.

Cell lines and culture conditions. Human GC MGC803 (low ADAM29 expression) and AGS (high ADAM29 expression) cell lines were purchased from American Type Culture Collection (Manassas, VA, USA). These cells were incubated with RPMI-1640 supplemented with $1 \mathrm{X}$ penicillin/streptomycin and $10 \%$ fetal calf serum (Gibco, Thermo Fisher Scientific. Inc., Waltham, MA, USA), in an incubator at $37^{\circ} \mathrm{C}$ with an atmosphere containing $5 \% \mathrm{CO}_{2}$ and $95 \%$ humidity.

Plasmids and transfection. The Flag-ADAM29 plasmid and ADAM29-small interfering (si)RNA were purchased from Shanghai GeneChem Co., Ltd. (Shanghai, China). MGC803 cells were either transfected with $1 \mu \mathrm{g}$ Flag-ADAM29 or the control vector pCMV (GeneChem, Shanghai, China) respectively using the X-tremeGENE HP DNA Transfection Reagent (Roche Diagnostics GmbH, Mannheim, Germany). AGS were transiently transfected with $1 \mu \mathrm{g}$ ADAM29-siRNA and corresponding control vector GV112 (GeneChem) using the aforementioned transfection reagent. The si-ADAM29 sequence was: Forward 5'-CCGGGCACT CTGACTGATGGTTCTACTCGAGTAGAACCATCAGTC AGAGTGCTTTTTG-3', and reverse 5'-AATTCAAAA AGCACTCTGACTGATGGTTCTACTCGAGTAGAACCA TCAGTCAGAGTGC-3'. ADAM29 expression was verified using western blotting (described subsequently) following transfection for $48 \mathrm{~h}$.

Immunohistochemical (IHC) staining. All GC sections were dewaxed and rehydrated using routine methods (27), and incubated with $5 \%$ bovine serum albumin (GeneChem) blocking solution for $30 \mathrm{~min}$ at room temperature. Slides were probed with the ADAM29 antibody (1:200; Abnova, Taipei, Taiwan) or with PBS for the negative control. Following extensive washing three times, sections were incubated for $30 \mathrm{~min}$ with a peroxidase-conjugated goat anti-mouse $\mathrm{IgG}$ (1:200; TA130004, OriGene Technologies, Beijing, China) at room temperature. Following washing three times to remove any unbound secondary antibodies, the section color was developed with 3,3-diaminobenzidine chromogen (Cell Signaling Technology, Inc., Danvers, MA, USA). A light microscope with x10 and x20 magnification (BX43; Olympus Corporation, Tokyo, Japan) was used for observation and capturing images.

$R N A$ isolation and reverse transcription-quantitative polymerase chain reaction (RT-qPCR). Following the manufacturer's protocol, total RNA isolated from the GC samples with the ABgene Total RNA Isolation Reagent (Advanced Biotechnologies Ltd., Epsom, Surrey, UK). cDNA was generated from $1 \mu \mathrm{g}$ of each RNA sample using GoScript ${ }^{\mathrm{TM}}$ Reverse Transcription system kit (Promega Corporation, Madison, WI, USA). ADAM29 mRNA level was quantified by 
Table I. Association between ADAM29 mRNA and clinical parameters.

\begin{tabular}{lccc}
\hline Category & $\mathrm{N}$ & Median & IQR \\
\hline Tissue sample & & & $<0.001-3.095$ \\
$\quad$ Normal & 25 & $<0.001$ & $<0.001-30.737$ \\
$\quad$ Tumor & 83 & 0.635 & \\
Sex & 52 & & $<0.001-26.377$ \\
Male & 31 & 0.693 & $<0.001-15.562$ \\
Female & & 0.572 & \\
Tumor grade (27) & 16 & & $<0.001-0.531$ \\
1 & 29 & 0.359 & $<0.001-13.127$ \\
2 & 38 & 0.725 & $0.353-22.772$ \\
3 & & 4.397 & 0.345 \\
TNM staging & 19 & & $<0.001-0.317$ \\
I & 27 & $<0.001$ & $<0.001-15.185$ \\
II & 37 & 0.816 & $<0.001-32.146$ \\
III and IV & & 5.447 & $<0.001-17.172$ \\
Location & 27 & 0.158 & $<0.001-25.398$ \\
Cardia & 56 & 0.698 & 0.572 \\
Non-cardia & & & $0.038^{\mathrm{a}}$ \\
\hline
\end{tabular}

${ }^{a} \mathrm{P}<0.05$. IQR, interquartile range; TNM, Tumor-Node-Metastasis. P-values are expressed as a comparison between the means using non-paired Student's t-test. ADAM metallopeptidase domain 29.

RT-qPCR using a QuantiNova SYBR Green PCR kit (Qiagen $\mathrm{GmbH}$, Hilden, Germany). Data was analyzed using the $2^{-\Delta \Delta \mathrm{Ct}}$ method (28). The qPCR primers were as follows: ADAM29 forward, 5'-CAGAGGCATGACACCTCCAG-3'; and reverse, 5'-TGGACAAATGGCTGGTCCTC-3'; $\beta$-actin forward, 5'-CTGGACTTCGAGCAAGAGATG-3'; and reverse, 5'-GAG TTGAAGGTAGTTTCGTGGA-3'. qPCR was programmed as followed: $95^{\circ} \mathrm{C}$ for $15 \mathrm{~min}$, then 60 cycles of $95^{\circ} \mathrm{C}$ for $20 \mathrm{sec}$, $55^{\circ} \mathrm{C}$ for $30 \mathrm{sec}$ and $72^{\circ} \mathrm{C}$ for $20 \mathrm{sec}$.

Western blot analysis. To investigate the expression level of ADAM29 in the MGC803 and AGS cells, confluent cells were centrifuged at $2,400 \mathrm{x}$ g for $10 \mathrm{~min}$ at $4^{\circ} \mathrm{C}$ and lysed with lysis buffer (Beyotime Institute of Biotechnology, Shanghai, China). The protein concentration was measured with a bicinchoninic acid protein assay kit (Beijing ComWin Biotech Co., Ltd., Beijing, China). A total of $5 \mu \mathrm{g} 20 \mu \mathrm{l}$ proteins were separated with $10 \%$ SDS-PAGE and blotted on to a nitrocellulose membrane. Following protein transfer, the membrane was treated with $5 \%$ skimmed milk to block non-specific proteins for $1 \mathrm{~h}$ at room temperature. Specific proteins were separately probed with the aforementioned anti-ADAM29 primary antibody (1:1,000; Abnova, Taipei, Taiwan) and anti-GAPDH antibody (1:1,000; cat no. sc-32233; Santa Cruz Biotechnology, Inc., Dallas, TX, USA) overnight at $4^{\circ} \mathrm{C}$, and then followed by incubation with aforementioned peroxidase-conjugated secondary antibody (1:200) for $1 \mathrm{~h}$ at room temperature. Protein bands were visualized using the Tanon High-sig ECL (Tanon Science and Technology Co., Ltd., Shanghai, China) and analyzed with Vilber Fusion Fx5 Spectra (Vilber Lourmat, Marne La Vallée, France).
In vitro cell growth assay. MGC803 and AGS Cell suspensions were seeded into 96 -well plates $(3,000$ cells/200 $\mu \mathrm{l} /$ well). The growth of the cells was assessed following a period of incubation ( $\leq 5$ days) in quadruplicate (overnight, day 3 , day 4 and day 5) in the previously stated conditions. Following incubation, the medium was removed and cultured with 10\% Cell Counting Kit-8 (Dojindo Molecular Technologies, Inc., Kumamoto, Japan) for $1 \mathrm{~h}$ at $37^{\circ} \mathrm{C}$. Subsequently, the absorbance was determined at the wavelength of $450 \mathrm{~nm}$ using a spectrophotometer (BioTek Instruments, Inc., Winooski, VT, USA).

In vitro cell wound assay. MGC803 and AGS Cells (1x10 $/ 600 \mu \mathrm{l} /$ well) were added into 12 -well plates and cultured overnight at $37^{\circ} \mathrm{C}$ to form a confluent monolayer. Subsequently, an artificial wound was produced in the monolayer with a $200 \mu \mathrm{l}$ pipette tip and washed twice with PBS to remove floating cells. The migration of cells was traced and recorded every $6 \mathrm{~h}$ by an inverted microscope at $\mathrm{x} 10$ and $\mathrm{x} 20$ magnification for $24 \mathrm{~h}$. The wound was measured and analyzed using ImageJ software (version 1.62; National Institute of Health, Bethesda, MD, USA).

In vitro invasion assay. Transwell chambers (upper inserts) polycarbonate filter inserts (BD Biosciences, Franklin Lakes, NJ, USA) with $8-\mu \mathrm{m}$ pore size were coated with $50 \mu \mathrm{g}$

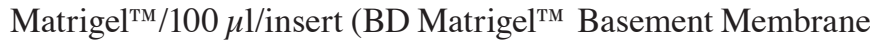
Matrix; BD Biosciences) and air-dried overnight at room temperature. Following rehydration, 20,000 cells/200 $\mu \mathrm{l} /$ insert with RPMI-1640 supplemented with $5 \%$ fetal calf serum (Gibco; Thermo Fisher Scientific, Inc.) were seeded in inserts 
A

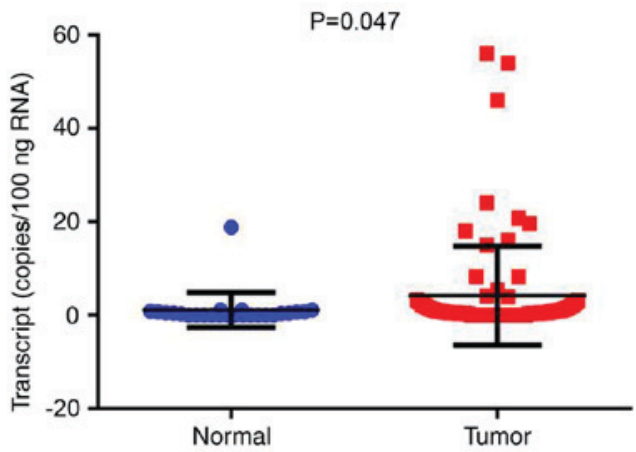

B

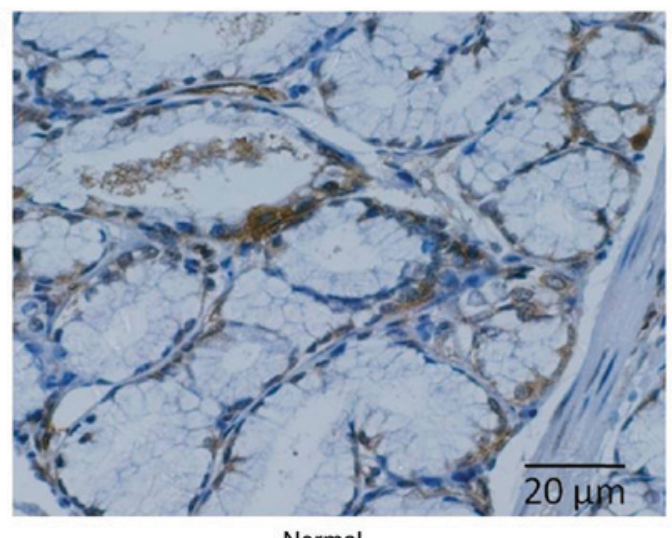

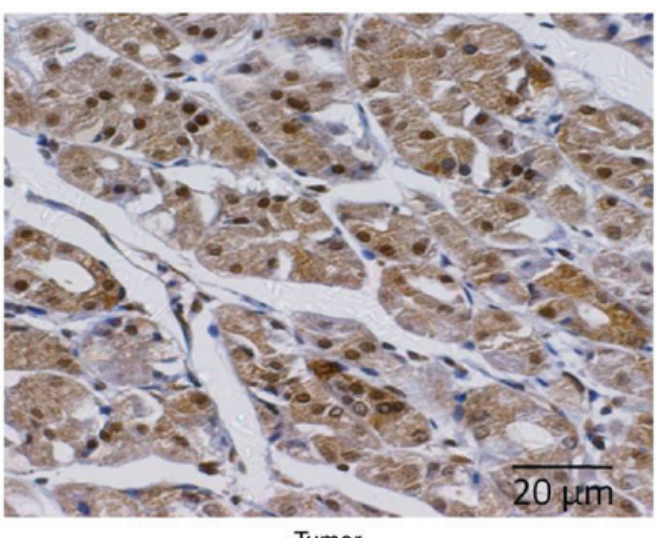

Tumor

Figure 1. (A) Expression of ADAM29 in tumor and paracancerous tissues of patients with GC. The ADAM29 transcription level was increased in human GC samples. (B) Immunohistochemical staining of ADAM29 in normal gastric tissue (left panel) and tumor tissue (right panel). Error bars represent the lower quartile range. GC, gastric cancer; ADAM29, ADAM metallopeptidase domain 29.

and $1 \mathrm{ml}$ RPMI-1640 medium with $10 \%$ fetal calf serum (Gibo; Thermo Fisher Scientific, Inc.) in the lower chamber for $72 \mathrm{~h}$ at $37^{\circ} \mathrm{C}$. Following incubation, cells that had invaded through the Matrigel ${ }^{\mathrm{TM}}$ and insert membrane and adhered to the other side of the inserts were fixed with $4 \%$ formalin for $20 \mathrm{~min}$ and stained with $0.5 \%$ crystal violet for $20 \mathrm{~min}$ at room temperature. Subsequently, crystal violet staining was dissolved with $10 \%$ acetic acid and measured at the wavelength of $570 \mathrm{~nm}$ spectrophotometer (BioTek Instruments, Inc.).

Statistical analysis. Data are presented as the mean \pm standard deviation. Experimental operations were repeated independently $>3$ times. Normally-distributed data were assessed using non-paired (two-sided) Student's t-test for two groups, whilst the Mann-Whitney U test was used for non-parametric distribution. Kaplan-Meier analysis was used to detect the association between ADAM29 expression and survival time of patients with GC (KM plotter; http://kmplot.com/analysis/).

\section{Results}

ADAM29 expression is associated with the clinical stages of $G C$. The expression pattern of ADAM29 was examined in 108 tissue specimens from patients with GC using RT-qPCR, including $83 \mathrm{GC}$ tissues and 25 adjacent normal tissues. The association between the expression level of ADAM29 and clinicopathological features is summarized in Table I. In the analysis of ADAM29 gene expression array data of the human GC tissue specimens, a significantly increased level of ADAM29 transcripts was observed in GC tumor sections compared with the adjacent normal gastric sections $(\mathrm{P}=0.047$; Fig. 1A). A significantly increased level of ADAM29 transcripts was observed in patients with GC and TNM III or IV stages compared with patients with TNM I stage ( $\mathrm{P}=0.038)$. Furthermore, an increased level of ADAM29 was determined in patients with terminal stage disease when compared with tumor grade I; however, it was not statistically significant (Table I).

In addition, the expression pattern of ADAM29 at the protein level also demonstrated similar results to its expression at the mRNA level in the IHC assay (Fig. 1B). It was determined that ADAM29 was expressed in the cytoplasm of normal gastric epithelial cells, and in cancerous cells. The carcinoma specimens demonstrated notably increased protein expression of ADAM29, whilst the paired paracancerous tissues consistently indicated weak or undetectable immunostaining.

Expression of ADAM29 is associated with the survival of patients with $G C$. To additionally study the functional aspects of ADAM29 in GC, the association between ADAM29 expression (Affy ID 221337_s_at) and the survival period of patients with GC was determined using KMplot. The Kaplan-Meier survival curves demonstrated that patients with GC and a low ADAM29 transcript level exhibited a significantly longer overall survival (OS) time when compared with patients with a high ADAM29 transcript ( $\mathrm{P}<0.001$; Fig. 2A), in a cohort of 882 cases of GC. In addition, decreased ADAM29 expression was also significantly associated with a reduced progression-free survival (PFS) of a cohort of 646 samples (P<0.001; Fig. 2B). 

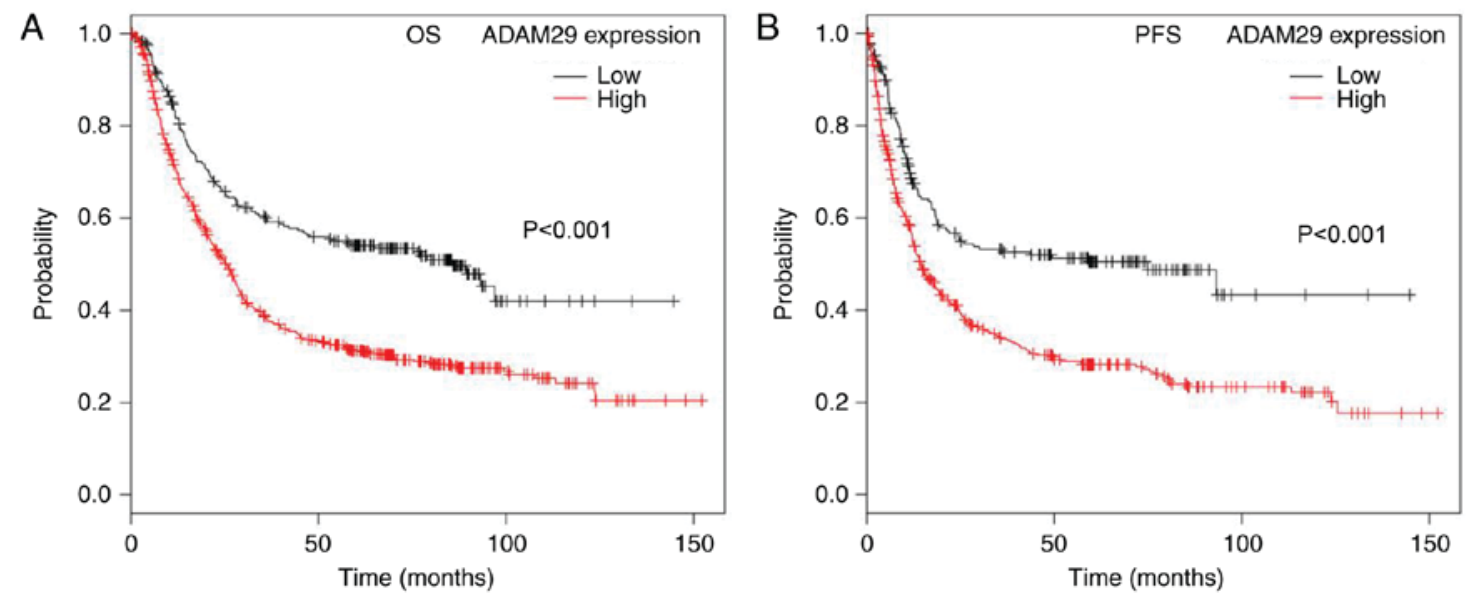

Figure 2. ADAM29 expression and survival of patients with GC. Association between ADAM29 expression and (A) OS and (B) PFS (both P<0.001) time was analyzed using the online Kaplan-Meier survival analysis (KMplot, http://kmplot.com/analysis/). GC, gastric cancer; OS, overall survival; PFS, progression-free survival; ADAM29, ADAM metallopeptidase domain 29.

A

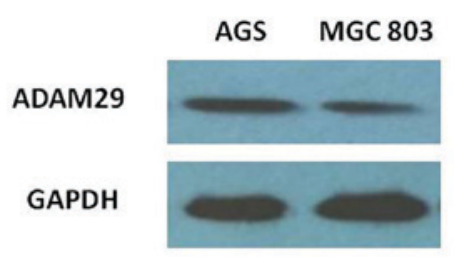

B
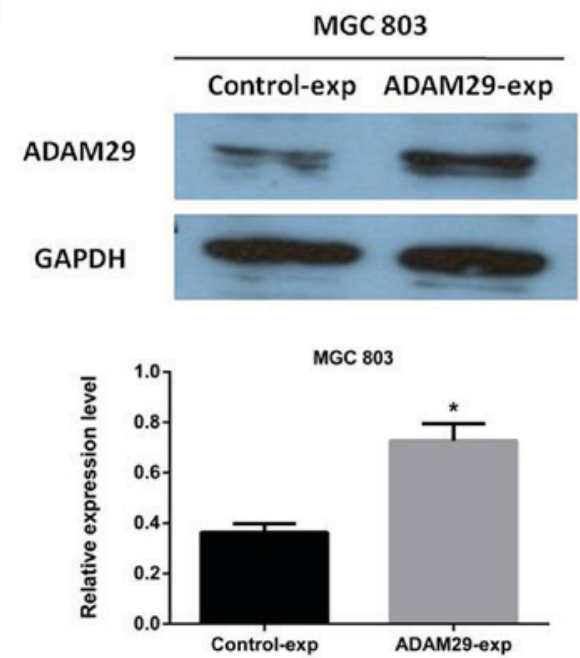

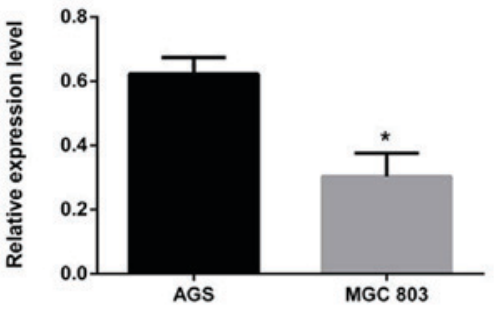

C
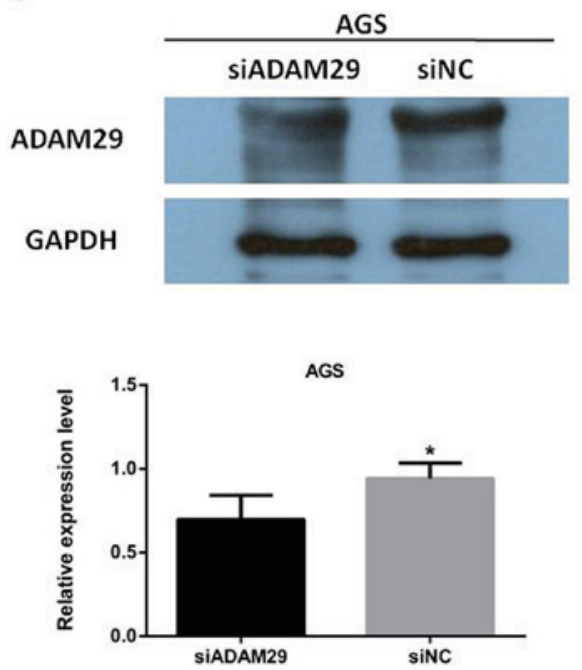

Figure 3. Expression level of ADAM29 in GC cell lines. (A) Expression of ADAM29 in MGC803 and AGS cells was determined using western blot analysis. (B) Following transfection, overexpression of ADAM29 in MGC803 GC cells and (C) knockdown of ADAM29 in AGS GC cells at the protein level were determined using western blot analysis. *P<0.05. ADAM29, ADAM metallopeptidase domain 29; GC, gastric cancer; exp, overexpressed; si, small interfering.

Expression level of ADAM29 in GC cell lines. To select suitable cell lines for biological function of GC cells, the expression of ADAM29 in AGS and MGC803 cells was analyzed using western blotting. It was determined that ADAM29 was highly expressed in AGS cells and exhibited low expression in MGC803 cells (Fig. 3A). Following plasmid and siRNA transfection in MGC803 and AGS cells, respectively, it was confirmed that the expression level of ADAM29 was considerably increased in the MGC803 cells (Fig. 3B), whilst there was a decreased ADAM29 expression level in the AGS cells (Fig. 3C). Graphical quantification for the overexpressed and knockdown levels of ADAM29 identified using western blotting is presented in Fig. 3 .

Effect of ADAM29 on GC cells growth in vitro. Compared with the control group, increased ADAM29 expression resulted in a significant increase in the growth of MGC803 cells (Fig. 4A; $\mathrm{P}<0.01$ ), whereas the knockdown of ADAM29 

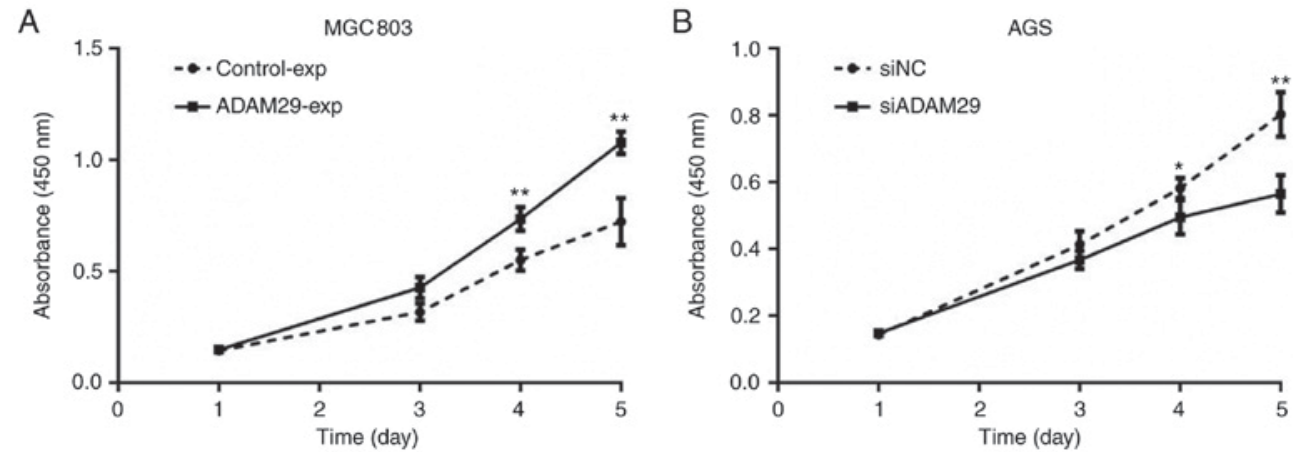

Figure 4. ADAM29 increases GC cell growth. (A) ADAM29 overexpression increased MGC803 cell growth compared with the control group in a time-dependent manner. (B) A decreased growth rate of cells was determined in ADAM29-knockdown AGS cells compared with the control cells ("P $<0.05$, ${ }^{* *} \mathrm{P}<0.01$ vs. control group). NC, negative control; ADAM29, ADAM metallopeptidase domain 29; exp, overexpressed; si, small interfering. GC. gastric cancer.

A
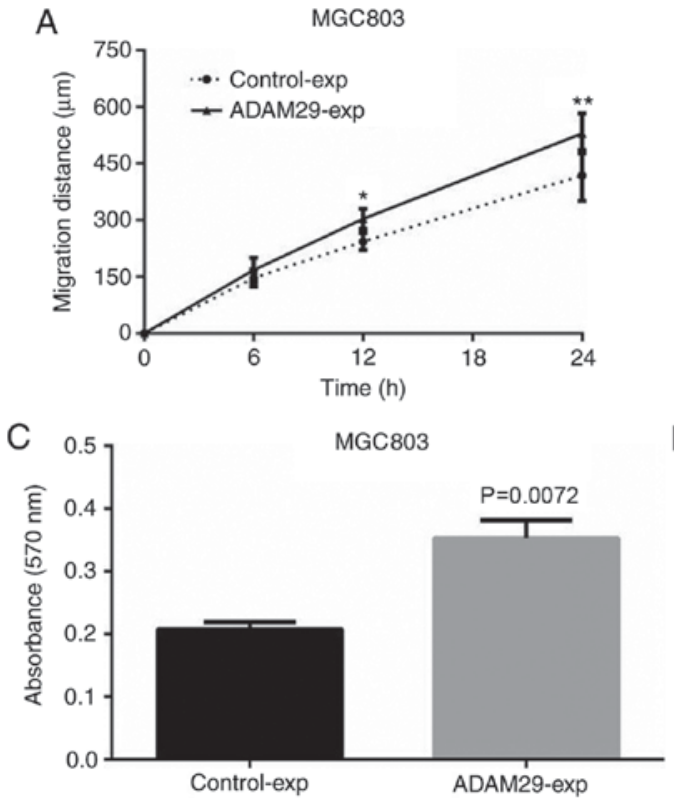

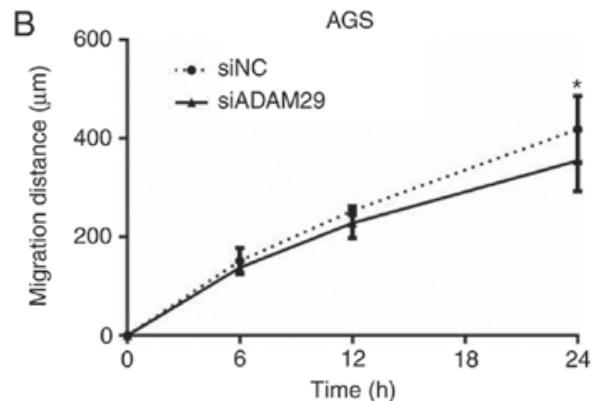

D $0.4 \quad$ AGS

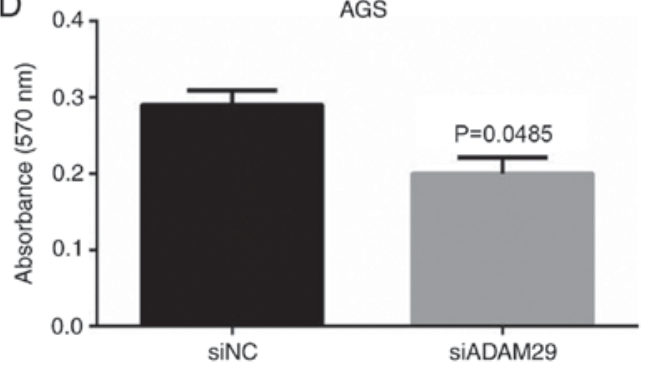

Figure 5. ADAM29 improves the migration and invasion of GC cells in vitro. (A) Increased migration rate was determined in ADAM29-overexpressing MGC803 cells. (B) Knockdown of ADAM29 in AGS cells decreased the GC cell migration distance. (C) ADAM29 overexpression increased MGC803 cell invasion compared with the control group. (D) A decreased invasion rate of cells was observed in ADAM29-knockdown AGS cells compared with the control cells ( $\mathrm{P}<0.05$ or $^{* *} \mathrm{P}<0.01$ vs. control group). ADAM29, ADAM metallopeptidase domain 29; GC, gastric cancer; NC, negative control; exp, overexpressed; si, small interfering.

caused a significant decrease in the growth ability of AGS cells (Fig. 4B; P<0.01). These results demonstrated that ADAM29 promoted GC cell growth.

Effect of ADAM29 on GC cells invasion and migration. Migration and invasion are important components of the malignant phenotype of cancer. The effect of ADAM29 on migration and invasion, respectively, was also assessed through wound healing and Transwell assays, respectively. Functional experiments in vitro indicated that the overexpression of ADAM29 promoted MGC803 cell migration (Fig. 5A; $\mathrm{P}<0.01)$ and knockdown of ADAM29 decreased AGS cell migration (Fig. 5B; $\mathrm{P}<0.05$ ). The invasion assay demonstrated that, compared with the control groups, the overexpression of ADAM29 in MGC803 cells significantly promoted the invasion ability of GC cells (Fig. 5C; $\mathrm{P}=0.0072$ ), whereas knockdown of ADAM29 resulted in a significant reduction in the invasion ability of the AGS cells (Fig. 5D; $\mathrm{P}=0.0485$ ).

\section{Discussion}

ADAM29 has been frequently demonstrated to be associated with the progression of cancer (18-21). In the present study, it was determined that ADAM29 was upregulated in GC tissues compared with paracancerous tissues via RT-qPCR and IHC analyses. Furthermore, the expression of ADAM29 was demonstrated to be associated with TNM staging of GC.

The important roles of ADAM family in the progression of various cancer types, including breast and gastric cancer, have been studied (16,25,28-30). For example, ADAM10 and ADAM17 may facilitate the migration of cancer stem cells resulting in a differentiated phenotype that is more susceptible to treatment (8). Previously, Micocci et al (29) identified that ADAM9 silencing inhibited breast cancer cell transmigration via blood and lymphatic endothelial cells. An additional study demonstrated that ADAM17 promoted the invasion, 
motility and sprouting of lymphatic endothelial cells (30). The anti-lymphangiogenic role of ADAM17 silencing in lymphatic endothelial cells indicated that ADAM17 may serve as a potential target in cancer therapy (30). The role of the ADAM family in GC has been studied previously $(16,25)$. Previously, a study determined that ADAM17 promoted epithelial-mesenchymal transition via activating the TGF- $\beta /$ Smad pathway in GC cells, which additionally indicated the relevance of ADAM17 as a potential target in cancer therapy (16). ADAM29 expression also affected the malignant phenotype of tumor cells (25). For example, ADAM29 overexpression significantly increased the proliferation, migration and invasion of breast cancer cells in vitro (22). Furthermore, it has been demonstrated that the mutation of ADAM29 frequently occurs in cancer (31). It was indicated that ADAM29 may serve a pivotal role in the process of breast cancer progression and metastasis (22). An additional study indicated that somatic mutations of ADAM29 frequently occurred in melanoma (19); however, the molecular mechanism underlying ADAM29 in GC remains unclear. To clarify the function of ADAM29, 7 tumor-derived mutants were created in melanoma cells, and its effect on growth and adhesion was examined in a previous study (19). In the present study, the focus was on the functional aspects of ADAM29 in mediating the malignant phenotype of GC cells. The present study determined that the overexpression of ADAM29 is associated with the elevation of growth, migration and invasion properties of MGC803 in vitro. Concomitantly, a significant decrease in the cellular migratory, proliferative and invasive abilities was identified in AGS cells when ADAM29 was knocked down, when compared with the control group. In combination, these data indicated the pro-cancer role of ADAM29 in GC cells; however, whether mutated ADAM29 may affect these malignant phenotypes, including proliferation, migration and invasion of GC cells is yet to be investigated. In future studies, the complete role of the ADAM29 mutation in GC will be explored.

The effect of ADAM29 on patient survival is not a negligible factor. Kaplan-Meier survival analyses of ADAM29 transcripts in GC indicated that increased levels of ADAM29 expression was were associated with poor OS and PFS time of patients with GC, suggesting that ADAM29 promoted disease progression and relapse of GC. In future studies, clinical outcomes will be measured to reveal the implications of ADAM29 in patient survival.

In conclusion, the data of the present study demonstrated that increased levels of ADAM29 were associated with poorer clinical outcomes of patients with GC. In vitro cell function data indicated that ADAM29 served as an oncogene in GC cells. These data indicated that ADAM29 may serve as a molecular marker in GC diagnosis and as an indicator for prognosis.

\section{Acknowledgements}

Not applicable.

\section{Funding}

The work was supported by foundation of Yantai Yuhuangding Hospital and Yantaishan Hospital (grant no. YT2017008).

\section{Availability of data and materials}

All data generated or analyzed during this study are available from the corresponding author on reasonable request.

\section{Authors' contributions}

HC designed the research; HC and SW performed the experiments; HC and SW contributed to writing and revision of the manuscript; all authors read and approved final manuscript.

\section{Ethics approval and consent to participate}

The present study was executed accordingly under the protocol approved by the Institutional Review Board and Research Ethical Committee of the Affiliated Yantai Yuhuangding Hospital of Qingdao University (Yantai, China). Written informed consent was obtained from all patients.

\section{Consent for publication}

All patients provided their written informed consent for the publication of their data.

\section{Competing interests}

The authors declare that they have no competing interests.

\section{References}

1. Ferlay J, Soerjomataram I, Dikshit R, Eser S, Mathers C, Rebelo M, Parkin DM, Forman D and Bray F: Cancer incidence and mortality worldwide: Sources, methods and major patterns in GLOBOCAN 2012. Int J Cancer 136: E359-E386, 2015.

2. Torre L, Bray F, Siegel R, Ferlay J, Lortet-Tieulent J and Jemal A: Global cancer statistics, 2012. CA Cancer J Clin 65: 87-108, 2015.

3. Hu Y, Huang C, Sun Y, Su X, Cao H, Hu J, Xue Y, Suo J, Tao K, He X, et al: Morbidity and mortality of laparoscopic versus open D2 distal gastrectomy for advanced gastric cancer: A randomized controlled trial. J Clin Oncol 34: 1350-1357, 2016.

4. Irani S, Baron T, Itoi T and Khashab M: Endoscopic gastroenterostomy: Techniques and review. Curr Opin Gastroenterol 33: 320-329, 2017.

5. Zhu T, Hu X, Wei P and Shan G: Molecular background of the regional lymph node metastasis of gastric cancer. Oncol Lett 15: 3409-3414, 2018

6. Walkiewicz K, Gętek M, Muc-Wierzgoń M, Kokot T and Nowakowska-Zajdel E: The importance of ADAM family proteins in malignant tumors. Postepy Hig Med Dosw (Online) 70: 67-73, 2016 (In Polish).

7. Brown RV, Gaerig VC, Simmons T and Brooks TA: Helping Eve overcome ADAM: G-quadruplexes in the ADAM-15 promoter as new molecular targets for breast cancer therapeutics. Molecules 18: 15019-15034, 2013.

8. Pham D, Kim J, Kim S, Shin D, Uong N, Hyun H, Yoon MS, Kang SJ, Ryu YJ, Cho JS, et al: Effects of ADAM10 and ADAM17 inhibitors on natural killer cell expansion and antibody-dependent cellular cytotoxicity against breast cancer cells in vitro. Anticancer Res 37: 5507-5513, 2017.

9. Edwards DR, Handsley MM and Pennington CJ: The ADAM metalloproteinases. Mol Aspects Med 29: 258-289, 2008.

10. Qin H, Diaz A, Blouin L, Lebbink R, Patena W, Tanbun P, LeProust EM, McManus MT, Song JS and Ramalho-Santos M: Systematic identification of barriers to human iPSC generation. Cell 158: 449-461, 2014.

11. Nyren-Erickson E, Jones J, Srivastava D and Mallik S: A disintegrin and metalloproteinase-12 (ADAM12): Function, roles in disease progression, and clinical implications. Biochim Biophys Acta 1830: 4445-4455, 2013. 
12. Wolfsberg T, Straight P, Gerena R, Huovila A, Primakoff P, Myles D and White JM: ADAM, a widely distributed and developmentally regulated gene family encoding membrane proteins with a disintegrin and metalloprotease domain. Dev Biol 169: 378-383, 1995 .

13. Ohtsuka T, Shiomi T, Shimoda M, Kodama T, Amour A, Murphy G, Ohuchi E, Kobayashi K and Okada Y: ADAM28 is overexpressed in human non-small cell lung carcinomas and correlates with cell proliferation and lymph node metastasis. Int J Cancer 118: 263-273, 2006.

14. Le Pabic H, Bonnier D, Wewer U, Coutand A, Musso O, Baffet G, Clément B and Théret N: ADAM12 in human liver cancers: TGF-beta-regulated expression in stellate cells is associated with matrix remodeling. Hepatology 37: 1056-1066, 2003.

15. Yin H, Zhong F, Ouyang Y, Wang Q, Ding L and He S: Upregulation of ADAM12 contributes to accelerated cell proliferation and cell adhesion-mediated drug resistance (CAM-DR) in Non-Hodgkin's Lymphoma. Hematology 22: 527-535, 2017.

16. Xu M, Zhou H, Zhang C, He J, Wei H, Zhou M, Lu Y, Sun Y, Ding JW, Zeng J, et al: ADAM17 promotes epithelial-mesenchymal transition via TGF- $\beta /$ Smad pathway in gastric carcinoma cells. Int J Oncol 49: 2520-2528, 2016.

17. Cerretti D, DuBose R, Black R and Nelson N: Isolation of two novel metalloproteinase-disintegrin (ADAM) cDNAs that show testis-specific gene expression. Biochem Biophys Res Commun 263: 810-815, 1999.

18. Song Y, Li L, Ou Y, Gao Z, Li E, Li X, Zhang W, Wang J, Xu L, Zhou Y, et al: Identification of genomic alterations in oesophageal squamous cell cancer. Nature 509: 91-95, 2014.

19. Wei X, Moncada-Pazos A, Cal S, Soria-Valles C, Gartner J, Rudloff U, Lin JC; NISC Comparative Sequencing Program, Rosenberg SA, López-Otín C and Samuels Y: Analysis of the disintegrin-metalloproteinases family reveals ADAM29 and ADAM7 are often mutated in melanoma. Hum Mutat 32 E2148-E2175, 2011.

20. Zhang B, Li Y, Li L, Chen M, Zhang C, Zuo X, Zhou FS, Liang B, Zhu J, Li P, et al: Association study of susceptibility loci with specific breast cancer subtypes in Chinese women. Breast Cancer Res Treat 146: 503-514, 2014.

21. Purrington K, Slager S, Eccles D, Yannoukakos D, Fasching P, Miron P, Carpenter J, Chang-Claude J, Martin NG, Montgomery GW, et al: Genome-wide association study identifies 25 known breast cancer susceptibility loci as risk factors for triple-negative breast cancer. Carcinogenesis 35: 1012-1019, 2014.
22. Zhao M, Jia W, Jiang W, Wang P, DU G, Cheng S and Song M: ADAM29 expression in human breast cancer and its effects on breast cancer cells in vitro. Anticancer Res 36: 1251-1258, 2016.

23. Brim H, Abu-Asab M, Nouraie M, Salazar J, Deleo J, Razjouyan H, Mokarram P, Schaffer AA, Naghibhossaini F and Ashktorab H: An integrative CGH, MSI and candidate genes methylation analysis of colorectal tumors. PloS One 9: e82185, 2014.

24. Ashktorab H, Schäffer A, Daremipouran M, Smoot D, Lee E and Brim H: Distinct genetic alterations in colorectal cancer. PloS One 5: e8879, 2010.

25. Costa N, Paulo P, Caffrey T, Hollingsworth M and Santos-Silva F: Impact of MUC1 mucin downregulation in the phenotypic characteristics of MKN45 gastric carcinoma cell line. PloS One 6: e26970, 2011.

26. Kim SG and Hwang SH: The association between the duration of fluoropyrimidine-based adjuvant chemotherapy and survival in stage II or III gastric cancer. World J Surg Oncol 14: 102, 2016.

27. Yu H, Ye L, Mansel RE, Zhang Y and Jiang WG: Clinical implications of the influence of Ehm 2 on the aggressiveness of breast cancer cells through regulation of matrix metalloproteinase-9 expression. Mol Cancer Res 8: 1501-1512, 2010.

28. Livak KJ and Schmittgen TD: Analysis of relative gene expression data using real-time quantitative PCR and the 2(-delta delta $\mathrm{C}(\mathrm{T})$ ) method. Methods 25: 402-408, 2001.

29. Micocci KC, Moritz MN, Lino RL, Fernandes LR, Lima AG, Figueiredo CC, Morandi V and Selistre-de-Araujo HS: ADAM9 silencing inhibits breast tumor cells transmigration through blood and lymphatic endothelial cells. Biochimie 128-129: 174-182, 2016.

30. Mėżyk-Kopeć R, Wyroba B, Stalińska K, Próchnicki T, Wiatrowska K, Kilarski WW, Swartz MA and Bereta J: ADAM17 promotes motility, invasion, and sprouting of lymphatic endothelial cells. PLoS One 10: e0132661, 2015.

31. Motycková M, Zák P, Vroblová V, Andrýs C, Belada D, Malý J and Smolej L: Prognostic markers in chronic lymphocytic leukemia. Vnitr Lek 57: 847-857, 2011.

This work is licensed under a Creative Commons Attribution-NonCommercial-NoDerivatives 4.0 International (CC BY-NC-ND 4.0) License. 